\title{
¿CÓMO INTERVIENEN LOS USUARIOS EN EL ESPACIO PÚBLICO?
}

\author{
HOW DO USERS INTERVENE \\ IN PUBLIC SPACE?
}

RODRIGO TORNERO OPFERMANN

Universidad de Lima
El espacio público como área social, que invita a los usuarios al desarrollo de múltiples actividades, es también un territorio utópico de posibilidades donde se imaginan sueños y mejoras para la sociedad. La relación entre el espacio público y sus habitantes determina los factores que conducen a su transformación desde una base utópica. Dado que el espacio público de la ciudad informal no responde adecuadamente a las necesidades que la sociedad presenta, el diseño participativo consciente, que toma en cuenta la opinión e ideales de quienes utilizan el espacio, permite obtener un producto que satisfaga las exigencias de manera eficaz.

espacio público, transformación, ciudad informal, diseño participativo
Recibido: 3 de junio del 2019

Aprobado: 6 de agosto del 2019

doi: 10.26439/limaq2020.n006.4825

The public space as a social area that invites users to the development of multiple activities, is also an utopian territory of possibilities, where dreams and improvements for society are imagined. The relationship between the public space and its inhabitants determines the factors that lead to its transformation from a utopian basis. Since the public space of the informal city does not adequately respond to the needs that society presents, the conscious participatory design, which takes into account the opinion and ideals of those who use the space, allows obtaining a product that satisfies demands effectively.

public space, transformation, informal city, participative design 


\section{METODOLOGÍA}

El presente estudio es el resultado de la lectura de artículos indexados en repositorios como Scopus, JSTOR, Scielo, relacionados a la intervención del espacio público. Para esto utilicé palabras clave como: espacio público, diseño participativo, apropiación; posteriormente, relacioné la teoría con ejemplos puntuales.

\section{IMPORTANCIA DEL PROCESO DE DISEÑO DEL ESPACIO PÚBLICO EN LA CIUDAD}

El espacio público es un área que comparten diferentes ciudadanos en un determinado lugar. Según Perez-Valencillos (2013), en este se desarrollan las expresiones comunitarias necesarias para reconstruir redes sociales y mejorar la calidad de vida en la ciudad. Por lo tanto, juega un papel importante en el desarrollo colectivo de una ciudad o pueblo. Mientras la sociedad evoluciona, también lo hacen sus necesidades; es así como aparecen cambios cada vez más significativos que determinan tanto la calidad formal como la continuidad del espacio. Del mismo modo, el diseño del espacio público cumple un papel importante en la transformación de las necesidades (Di Siena, 2011), pero ¿qué pasa cuando este no responde a las necesidades que presenta la sociedad?

Con el transcurso del tiempo surgen determinados espacios de interacción en la ciudad, que satisfacen deseos, necesidades y expectativas que la sociedad demanda; sin embargo, cada vez son más los casos que no cumplen el rol para el que fueron diseñados originalmente utilizándoselos para actividades diferentes. Esto demuestra que el espacio público no responde de manera adecuada a la demanda de la sociedad ya que estas transformaciones, por minúsculas que sean, conforman y evidencian la identidad del lugar en que se encuentra (Mazza, 2009).

Son pocos los casos en que los ciudadanos son parte del proceso de diseño de un espacio público en la producción de la ciudad. Por lo tanto, la ciudad informal se basa en transformaciones por intervención posterior, lo que se ve reflejado en la participación en el mantenimiento por parte de la misma comunidad. Esto sucede en el caso del movimiento Ocupa tu Calle, promovido por el observatorio ciudadano Lima Cómo Vamos, donde se interviene la ciudad a fin de generar mejores espacios públicos para el disfrute de los ciudadanos de Lima, mediante la recuperación de espacios de la ciudad en desuso (Lima Cómo Vamos, 2014).

\section{LA BÚSQUEDA DE GENERAR NUEVOS ESPACIOS}

Muchas veces no solo se trata de la construcción de algo material sino de un soporte para la necesidad de expresión de los ciudadanos, visto en algo tan simple como un mural con graffiti, que, según Barcia y Ramallo (2015), expresa mucho más allá de lo que a, simple vista, refleja la pintura. 
Existe una fuerte relación entre el espacio público y la dinámica social de un lugar. Jan Gehl (2006) en "Humanización del espacio urbano" menciona una dimensión de la ciudad y el urbanismo, la dimensión socializante, que va relacionada con el potencial de la integración de la comunidad humana donde prioriza la vida social antes que cualquier edificio y resalta la importancia de hacer atractivo el espacio público porque es ahí donde se desarrollan las diversas actividades sociales y desde donde se percibe la ciudad.

La regeneración urbana, como explican Robertz y Sykes (1999), implica el proceso de reconstrucción de lugares. En este proceso, iniciativas de regeneración se planifican para mejorar las condiciones físicas de los lugares, aumentar el crecimiento económico y sostenibilidad ambiental para facilitar una mejor vida social para las personas.

Existen problemas de diseño que los usuarios resuelven de manera precaria porque no se tuvieron en cuenta sus necesidades y preferencias en el proceso. Es así como, si un espacio cuenta con la calidad visual como para querer detenerse y observar, la gente del lugar buscará la forma de hacerlo al ubicar bancas u objetos para sentarse por su propia cuenta (figura 1). O cuando en lugar de ir por la vereda se forma un sendero en el césped que indica la huella del camino más recorrido por los lugareños que no se tuvo en cuenta en el diseño original del parque.

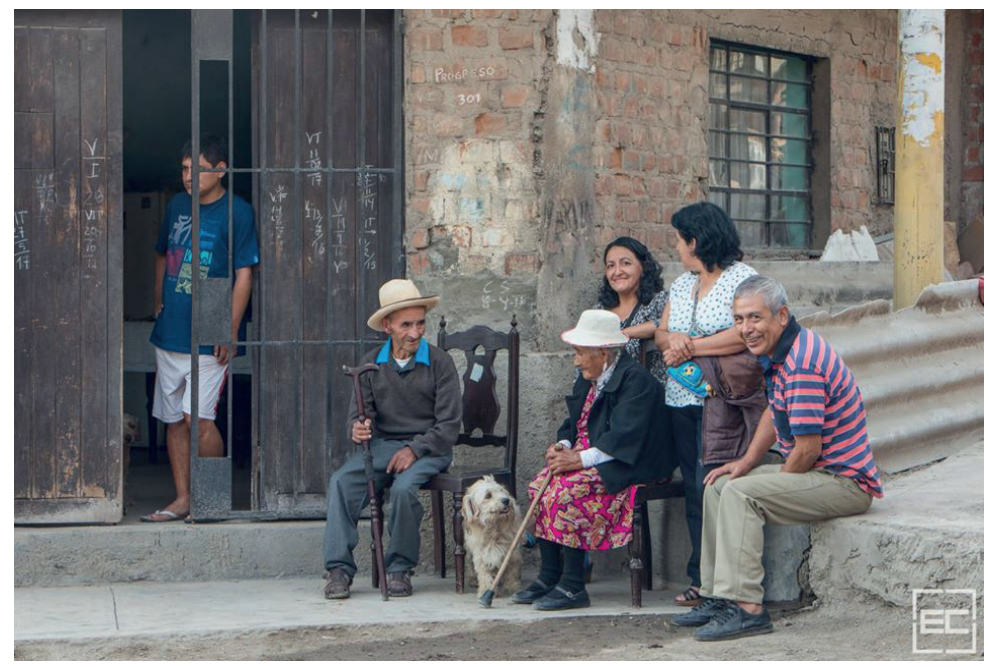

Figura 1.

Feriado familiar

Fuente:

Cuadros, 2018

\section{LA PERCEPCIÓN DEL USUARIO}

Como el concepto de lugar no es solo el de algo físico, sino psicológico, la percepción del usuario cumple un papel importante en la posible transformación del espacio. Para esto se debe tener en cuenta la opinión de personas 
en escenas reales de esta problemática, porque solo de esta manera podemos saber si se está cumpliendo a cabalidad con sus expectativas.

Cabe resaltar la importancia del sentido de pertenencia que se concreta a medida que avanza el uso y apropiación de espacios comunes por parte de los usuarios; por lo tanto, este conjunto de estímulos y sensaciones debe ser traducido a estrategias que ayuden a abordar el proceso de diseño del espacio (Carmona, 2016). Las personas hacen o sienten suyo un espacio debido a que este conforma una parte de su historia personal, esto determina un sentimiento de propiedad al cual se le otorga, según cada persona, un significado en base a sus necesidades y deseos (Rodríguez, 2014).

La percepción de la que hablo se puede dar con respecto a la ubicación del espacio, la proximidad a lugares de interés público o simplemente por el mal diseño de los ya construidos, que a opinión de los usuarios no satisfacen sus necesidades. Por otro lado, la percepción del usuario con respecto al lugar no solo se basa en estándares temporales, sino en el apego al contexto, el cual no necesariamente debe responder a una unidad de tiempo que defina el establecimiento de nuevos usuarios.

Sabemos, según Lewicka (2010), que cuando nuevas personas se asientan en un espacio natural, estos son considerados, al menos al inicio, extraños en la comunidad que residió ahí por generaciones. Esto es un fenómeno universal, hecho que se encuentra comprobado por la gran cantidad de apellidos que hacen referencia a "un recién llegado" como Newmann o Novak. A medida que la popularidad de un lugar aumenta, también lo hace la cantidad de nuevos visitantes, los cuales podrían pasar a convertirse en nuevos residentes; sin embargo, estos usuarios no perciben el lugar como "menos suyo" por el hecho de tener menos tiempo que los locales que han estado ahí por generaciones.

Por lo tanto, se puede concluir en que existe una sumatoria de procesos de apropiación, la cual determina si el espacio se vuelve exitoso o no al funcionar adecuadamente; sin embargo, este se encuentra en constante evolución, debido a que, como parte del apropiar implica el interactuar y, por lo tanto, el intervenir, se crea una relación directa en que, entre más se interviene hay más apego de por medio. Esta intervención puede ser espontanea, organizada o informal.

\section{INTERVENCIÓN ESPONTÁNEA DEL ESPACIO PÚBLICO}

Para adaptarse a la nueva sociedad, llena de usuarios con necesidades cambiantes, estos espacios se redefinen en su nuevo contexto y se transforman de tal manera que terminan siendo diferentes a su origen anterior. Aunque la ciudad establezca un terreno abierto, el uso de los espacios dentro de esta no es igualitario para el común de los grupos sociales que lo habitan, debido a la constante llegada de nuevos participantes con otras necesidades e ideas que manifestar (Uría, 2001). 
Las diferentes actividades, realizadas por diversos actores demuestran la fragilidad de un sistema de uso constante en el espacio público, casos generales señalan que el uso de espacios tiende a ser específico según género, edad y tiempo (Hernández, 2013). De esta manera, un parque no representa lo mismo para un niño, joven o adulto, debido a la variación de razones para su uso, que puede ir desde caminar o hacer deporte, encontrarse en un entorno social o simplemente por gusto, afición y comodidad.

Un claro ejemplo es la exposición "Post-it City (2008). Ciudades ocasionales", realizada en el Centro de Cultura Contemporánea de Barcelona (CCCB). Allí se evidencia la necesidad de crear espacios que respondan eficazmente a las necesidades de la sociedad mediante fotografías que muestran diferentes usos temporales sobre el territorio del espacio público de la ciudad informal convirtiéndolo en un escenario de expresión de la sociedad.

Otro ejemplo, sin ir tan lejos, es la Unidad vecinal n. ${ }^{\circ} 3$, donde los mismos usuarios intervienen el espacio de acuerdo a las demandas de la estación. Durante el verano se implementan grandes piscinas portátiles que son costeadas y utilizadas por los vecinos de la unidad. Otras veces incluso se cierran algunas calles o vías peatonales por determinadas reuniones, ferias gastronómicas o ceremonias religiosas, las cuales de alguna u otra manera ayudan a fortalecer los vínculos sociales entre los miembros de la comunidad y, por lo tanto, su identidad.

Por otro lado, es importante reconocer cómo se manifiesta una relación subjetiva entre una intervención espontánea y el apego al lugar, el cual no necesariamente es positivo. Es aquí donde cito de ejemplo al asentamiento humano Flor de Amancaes, el cual se ubica peligrosamente cerca de las lomas de Amancaes, una importante área de preservación natural. $\mathrm{Al}$ recorrerlo se pueden observar los distintos niveles de consolidación a lo largo del tiempo, tanto en viviendas como en espacio público. El diseño de este último nunca fue planteado como una prioridad y se ve reflejado en su calidad, donde más allá de una adecuada intervención, que pudo iniciar de manera espontánea, refleja una improvisación que con el transcurso de los años se ha ido consolidando, dando como resultado espacios que no reflejan ni la identidad del lugar, ni un aporte a la ciudad y que ponen en riesgo tanto a las personas como a las lomas.

También se reconoce que existen casos donde el apego se da por no tener otra opción donde asentarse, como lo es el de la ciudad informal en zonas de huaico o derrumbe. En dichos espacios se busca adecuar el sitio a las condiciones del lugar, muchas veces de manera forzada; y aun cuando termine en desastre, vuelve a ser reconstruido debido a que hay un apego de por medio.

\section{INTERVENCIÓN ORGANIZADA DEL ESPACIO PÚBLICO}

Si hablamos de soluciones duraderas, sin ser excesivamente costosas para las municipalidades, se encuentra la peatonalización. Por ejemplo, en varias calles de áreas centrales de Buenos Aires, Argentina, se ha aplicado esta estrategia, 
donde se incrementan los recorridos peatonales a expensas de los vehiculares (Perahia, 2007). Este tipo de procedimiento, previamente planeado, es necesario para obtener un modelo de ciudad más amable con los usuarios, debidamente acompañado de medidas integrales de movilidad, urbanismo y medio ambiente. Es indispensable que estos procedimientos sean progresivos y no perjudiquen la habitabilidad de los espacios potenciales del peatón para, así, evitar futuros errores de planificación estratégica (Chilla, 2017).

Por otro lado, como respuesta por parte del sector académico, se presenta el caso de La Balanza, en Comas, uno de los distritos más caóticos de Lima, donde gracias a la intervención de movimientos culturales en colaboración de los mismos vecinos de la zona y organizadores sociales busca constituirse como la capital cultural de Lima norte (Lara, 2016).

De este modo, se busca convertir la sede central de la Fiesta Internacional de Teatro en Calles Abiertas (Fiteca), que actualmente es una losa deportiva, en un espacio que promueva la libre expresión de artistas urbanos mediante la pintura, música, poesía, danza y teatro, entre otros. La propuesta de diseño es el resultado de referentes de la zona, por lo que se establecieron una serie de criterios generales con base en la observación de la vida cotidiana del barrio (Proyecta, 2015).

También aparecen organizaciones no gubernamentales con propuestas como Urban 95 que buscan experimentar con los espacios de tal manera que, por medio de intervenciones menores, se logre recuperar espacios y promover la aceptación y cuidado como resultado del previo análisis del barrio y otorgarles la posibilidad de obtenerlo como lo imaginan. Parte fundamental del desarrollo del proyecto es la inserción de equipo técnico y social en las dinámicas sociales existentes del lugar para poder comprender sus puntos de vista y participar del proceso urbano; de esta manera, hacer puente hacia la municipalidad y demás instituciones (Urban 95, 2018).

Figura 2.

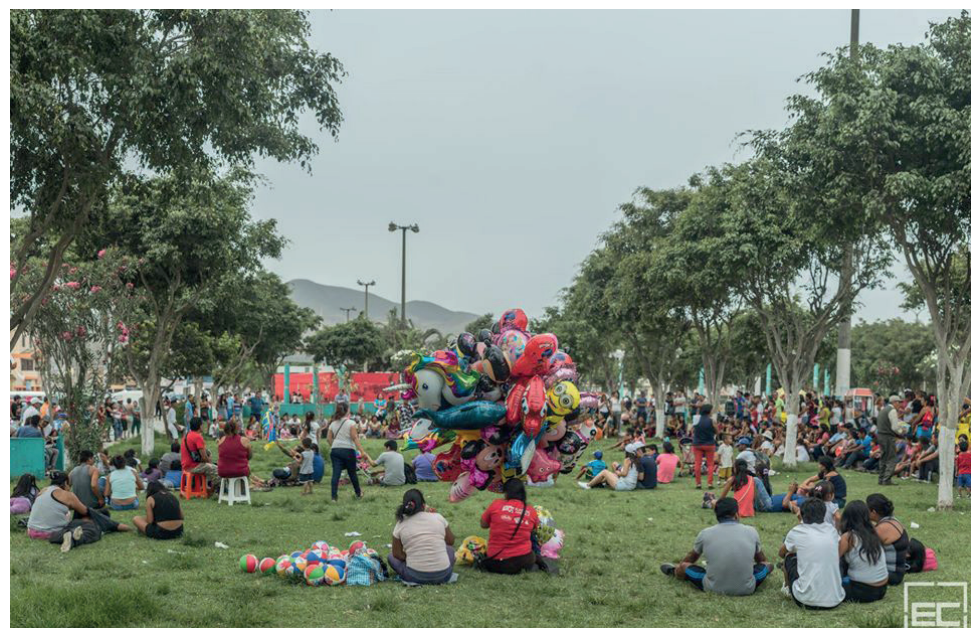


Tenemos el caso de Food Vibes Co. un equipo joven de amigos gastrónomos que cuentan con una flota de food trucks, con los que buscan generar cultura de comida urbana de calidad en Lima. Este grupo propone un concepto distinto que involucra la comida con actividades culturales, para esto se plantea la idea de contar con eventos en parques específicos del distrito cada fin de semana con un calendario de actividades donde los mismos vecinos se beneficien mejorando la calidad de vida de las comunidades de Lima al aprovechar al máximo los espacios públicos de la ciudad (figura 2). Se fomenta el desarrollo del espacio común de un distrito entre sus miembros teniendo como marco áreas verdes y propuestas artesanales de comida (Food Vibes, 2018).

\section{INTERVENCIÓN INFORMAL DEL ESPACIO FORMAL}

Si bien es cierto que existe una gran variedad de casos de alteraciones del espacio en zonas precarias de la sociedad, es importante saber que este tipo de manifestaciones no son ajenas a la ciudad formal, debido a que en su gran mayoría esta expresión apunta a la capacidad de flexibilizar la intervención urbanística y así lograr que los espacios no queden simplemente en suspensión por una adecuada urbanización, ya que el no encontrar utilidad a estos espacios con posibles propuestas capaces de activar aspectos sociales en la ciudad significaría una enorme pérdida (Fernández, 2012).

Estas intervenciones, además de la transitoriedad, se reconocen por su pequeña escala, buscan relacionarse con los ciudadanos, ya que mientras van activando espacios fomentan la participación entre usuarios y las relaciones sociales, pero en general, vienen motivadas por situaciones particulares, oponiéndose al diseño estándar y a gran escala de la ciudad. Son una serie de apropiaciones espontáneas del espacio relacionadas con el arte público o muestras arquitectónicas de pequeña y mediana escala que avanzan sin cuestionarse si tienen un impacto positivo o negativo en la ciudad. Pese a que muchas de estas prácticas $\mathrm{y}$ acciones ya se encuentren integradas al sistema se puede asumir que muchas de estas tienen algo de cuestionador, es decir, son un indicador de que algo no andaba bien en la ciudad (Sansão-Fontes, 2016).

También es importante reconocer estas intervenciones como oportunidades que amplían la posibilidad de disfrute de la misma ciudad que incluso puede llegar a generar ingresos temporales para el propio promotor; es una forma de crear utilidad social como parte de un proceso de transformación urbanístico complejo mediante la introducción de usos transitorios (Fernández, 2012).

Propongo como ejemplo la situación del Pentagonito en San Borja, que por lo general presenta un cálido y tranquilo ambiente familiar con espacios enfocados al deporte y el uso recreacional. Este lugar es conocido por la amplia variedad de actividades que se realizan en sus diferentes espacios. Por lo general, los fines de semana encontramos competencias caninas, food trucks, aeróbicos y baile, clases de yoga, artes marciales y hasta competencias de atletismo para las cuales, incluso, obstaculizan ciertas vías vehiculares para un mejor disfrute del lugar. 
Así como estas actividades son características importantes del Pentagonito, también lo es en lo efímero; es decir, con la rapidez que aparecen, desaparecen, llegando al día siguiente sin alguna prueba concreta de que sucedieron.

\section{APROPIACIÓN DEL ESPACIO}

Dentro de las características de las ciudades contemporáneas se encuentra la creación de espacios donde la delimitación de lo público sobrepasa las barreras establecidas por los parámetros, este es el caso de los centros comerciales, patios de comida, bares y restaurantes. Una idea de espacio público donde no se hace necesario pedir permiso para ingresar (Salazar, 2013).

Si hablo de la apropiación del espacio público, me refiero al acaparamiento de algo "ajeno" y la única forma de lograr esto es haciéndose el propietario de manera provisional a través de las mismas destinaciones de los mismos usuarios. Es así como, dentro de las características de estos espacios, se encuentra la posibilidad de ser privatizados por cualquier persona durante un tiempo limitado, restringiendo con su presencia el uso por parte de otro ciudadano en ese mismo momento (Salazar, 2013).

El problema está en que al ser de todos y a la vez de nadie, en él se presentan cada día múltiples identidades urbanas, las cuales se lo disputan a través de la conquista y el uso. Por lo tanto, se resalta lo importante que es reorganizar la sociedad por medio de referencias espaciales, es decir, sitios y lugares de convergencia para constituir propia y políticamente los espacios públicos de la ciudad (Almanda, 2014).

Como sucede en el parque Castilla en Lince, que pese a contar con una explanada y dos anfiteatros como parte de su infraestructura, no se da abasto para albergar a tantos grupos divididos, ya que cuando llegan personas a bailar, actuar o realizar algún tipo de evento, incluso ajenas al distrito, se produce un gran desorden que genera incomodidad entre los mismos usuarios; esto sumado a los ruidos molestos de los juegos mecánicos, comercio ambulatorio y equipos de sonido independientes crea un verdadero caos en el parque que ha llegado a molestar a los vecinos de la zona (Lara, 2016).

La apropiación también se dispone por un uso en particular del espacio. En muchos casos, este se determina como herramienta para llamar la atención de los usuarios que pasan por ahí, puede ser una forma de "sentirse vivos", o simplemente por una cuestión de necesidad económica como es el caso del comercio ambulatorio. Aun cuando estos ocupan parte del espacio público le están dando un uso, el cual es meramente económico, pero que también connota poder simbólico (Torres, 2009). El verdadero valor se encuentra en la transformación del espacio público mediante experiencias. Este puede ser utilizado desde movilización y entretenimiento hasta expresiones culturales y protesta ciudadana. Así es como se hace evidente el significado de la huella que se desarrolla en estos espacios y que servirá de soporte físico y cultural para promover una cultura de lo público (Burbano y Páramo, 2014). 
Durante el proceso de apropiación de escenarios urbanos se va forjando el sentido de pertenencia hacia el lugar conquistado presencial y simbólicamente, siendo el proceso de la construcción de identidades el mismo que se conforma desde las relaciones sociales producidas en dichos espacios, por lo tanto, también del sentido de exclusión (Almanda, 2014).

Cuando un asentamiento se encuentra en etapas iniciales y aún no cuenta con la infraestructura urbana adecuada, responde a un urbanismo de autoayuda. En este contexto, aparece una práctica de supervivencia y uso eficiente de escasos recursos disponibles a modo de construcción espontanea (Lara, 2014).

\section{DISCUSIÓN}

El apego al lugar no siempre implica un factor participativo positivo con respecto al desarrollo de un adecuado espacio público en la ciudad. Se debe considerar si realmente es una ventaja inicial el proceso de apropiación en zonas no consolidadas, como son aquellas junto a medios naturales.

En este punto es importante debatir el apego bajo una medición cualitativa (Lewicka, 2010), donde se priorice la importancia del lugar sobre su significado, esta graduación puede variar de acuerdo a la diferenciación entre personas con respecto a la importancia subjetiva y los vínculos emocionales que ello consiga. Sin embargo, implica un sesgo de información, debido a que existen diferentes "escalas de apego" que varían de acuerdo a la sensibilidad emocional con que cada persona percibe la dimensión de un lugar.

Sin embargo, con base en los casos y transformaciones del espacio público analizados se demuestra que lo que se hace sin consultar al ciudadano no logra la completa satisfacción de sus necesidades, debido a que no responde correctamente a las demandas de los ciudadanos.

Que el ciudadano sea parte del proceso de diseño traería grandes beneficios al desarrollo social de la ciudad ya que en estos aportes de primera mano se encuentran las principales necesidades de expresión y muestras de identidad. Es importante resaltar que aunque el espacio público haya sido diseñado para cumplir cierto objetivo, son los mismos usuarios los que a través de la rutina le otorgan su verdadera función y concepto.

No hace falta una construcción monumental como parte del proceso de la regeneración del espacio para promover en el usuario el sentido de pertenencia hacia el lugar. Basta con la integración de intervenciones de pequeña o mediana escala, como un mural con grafiti, relacionado a la dinámica social que reflejen la apropiación y sentido de identidad de los usuarios.

Existen distintos niveles de apropiación del espacio, los cuales no necesariamente se refieren a la transformación de este como tal; estos incluyen actividades como detenerse y pasar un momento sentado en la banca de la plaza, dejar 
una sombrilla en la playa o la construcción de un pequeño cerco alrededor del jardín. El espacio público hace una importante contribución a la identidad de la ciudad y al sentido de pertenencia de los ciudadanos al ser el centro de procesos de integración social.

\section{REFERENCIAS}

Almanda, H. (2014). La apropiación del espacio público a través de las prácticas deportivas juveniles. El Colegio de la frontera norte. Recuperado de https://www.colef.mx/posgrado/wp-content/uploads/2014/11/Tesis-AlmadaFlores.pdf

Barcia, S. y Ramallo, F. (2015). "Graffiti” y conflicto lingüístico: El paisaje urbano como espacio ideológico. Revista Internacional de Lingüistica Iberoamericana, pp.131-153. Recuperado de http://www.jstor.org/stable/24769005

Burbano, A. M. y Páramo, P. (2014). Los usos y la apropiación del espacio público para el fortalecimiento de la democracia. Revista de Arquitectura, 16, 6-15. http/dx.doi.org/10.14718/RevArq.2014.16.2

Cardona, B. (2008). Espacios de ciudad y estilos de vida El espacio público y sus apropiaciones. Revista Educación Física y Deporte. Recuperado de file://C:/Users/Rodrigo/Downloads/Dialnet-EspaciosDeCiudadYEstilosDe VidaEIEspacioPublicoYSus-3157873.pdf

Carmona, K. (2016). Espacio público como elemento generador de inclusión y cohesión social en la ciudad contemporánea latinoamericana. Recuperado de https:// upcommons.upc.edu/bitstream/handle/2117/80287/86BCN_Carmona Karen.pdf?sequence $=1$ \&isAllowed $=y$

Chilla, S. (2017). Peatonalizar para recuperar el espacio público. IU Jerez. Recuperado de http://iujerez.es/peatonalizar-para-recuperar-el-espacio-publico/

Cuadros, E. (2018). Espacio público limeño [fotografía]. Recuperado de https://web. facebook.com/presbiciadelandar/photos/p.2044927902421461/20449279 02421461/?type=1\&theater

Cuadros, E. (2018). Feriado familiar [fotografía]. Recuperado de https://web.facebook. com/presbiciadelandar/photos/a.1571435936437329/2080062205574697/ ?type=3\&theater

Di Siena, D. (5 de marzo del 2011). Ciudad sensible-espacio público. Entrevista a Juan López-Aranguren Blázquez. Urbanohumano. Recuperado de http:// urbanohumano.org/blog/2011/03/05/sentient-city-entrevista-a-efrain-foglia/

Fernández, M. (2012). El papel del urbanismo temporal en el master plan. Ciudades a escala humana. Recuperado de https://www.ciudadesaescalahumana. org/2012/09/usos-temporales-en-grandes-proyectos-de.html

Fernández, M. (2012). Urbanismo adaptativo. Attribution-ShareAlike License. Recuperado de https://es.slideshare.net/manuederra/urbanismo-adaptativo -la-ciudad-temporal-en-un-mientras-tanto-permanente-v2. 
Firouzmakan, S. y Abdolhadi, S. (2015). Promotion Quality of Life by Increasing Place Attachment in Public Places. Procedia - Social and Behavioral Sciences. Recuperado de: https://doi.org/10.1016/j.sbspro.2015.08.197

Food Vibes Co. (2018). Facebook. Recuperado de https://web.facebook.com/pg/ foodvibescompany/about?ref=page_internal

Francon, J. (2015). Espacios públicos amables para una ciudad informal, la experiencia de "Barrio Mío" en Lima. ArchDaily. Recuperado de https://www.archdaily. pe/pe/760924/espacios-publicos-amables-para-una-ciudad-informal-laexperiencia-de-barrio-mio-en-lima

Hernández, J. (2013). Construcción social de espacio público en barrios populares de Bogotá. Revista INVI, 28(78), pp.143-178.

La Balanza-Comas: Creación de barrios culturales. (2015). Proyecta. Recuperado de https://issuu.com/construccionyvivienda/docs/proyecta_edicion_33

Lara, E. (2014). Reconocimiento, uso y apropiación del espacio público en asentamientos informales. Recuperado de http://estudiosurbanos.uc.cl/ images/tesis/2014/MDU_Esther_Lara.pdf

Lara, J. (11 de julio del 2016). La Balanza: de zona roja a la capital cultural de Comas. El Comercio. Recuperado de https://elcomercio.pe/lima/balanza -zona-roja-capital-cultural-comas-235147

Lara, J. (3 de agosto del 2016). Lince: vecinos piden usar áreas verdes del parque Castilla. El Comercio. Recuperado de https://elcomercio.pe/lima/lincevecinos-piden-areas-verdes-parque-castilla-243680

Lewicka, M. (2010). Place Attachment: How far Have We Come in the Last 40 Years? ELSEVIER.

Lima Cómo Vamos. (2014). Ocupa tu calle. Recuperado de http://www. limacomovamos.org/ocupa-tu-calle/

Marín, L. (2015). Usos del espacio público y su influencia en la dinámica social del sector. La Esperanza del distrito de Chilca. Universidad Nacional del Centro del Perú. Huancayo.

Mazza, A. (2009). Ciudad y espacio público. Las formas de la inseguridad urbana. Cuaderno de Investigación Urbanística, 18(62).

Perahia, R. (2007). Las ciudades y su espacio público. Universidad de Buenos Aires.

Pérez-Valecillos, T. (2013). Creación del espacio público en asentamientos informales: nuevos desafíos urbanos. Bitácora23, p. 95-104.

Roberts, P.y Sykes, H. (1999). Urban Regeneration:AHandbook. Recuperadodehttps:// books.google.es/books? hl=es\&lr=\&id=oR7006MayFAC\&oi=fnd\&pg=PP1 $\& d q=$ Roberts $+y+$ Sykes\&ots=8VATfc82p_\&sig=upjH4aTtC_PFo6EO6N yTHmgXqIM\#v=onepage\&q =Roberts\%20y\%20Sykes\&f=false

Rodríguez, F. (2014). La importancia y la apropiación de los espacios públicos en las ciudades. Universidad de Guadalajara. Recuperado de: http://www. udgvirtual.udg.mx/paakat/index.php/paakat/article/view/222/329 
Salazar, J. (2013). Uso y apropiación de los espacios públicos. Issuu. Recuperado de https://issuu.com/salgadodigital/docs/uso_y_apropiacion_de_los_ espacios_publicos

Sansão-Fontes, A. (2016). Más allá de lo público y lo privado. Intervenciones temporales y creación de espacios colectivos en Río de Janeiro. Universidad Católica de Colombia. Recuperado de https://editorial.ucatolica.edu.co/ojsucatolica/ revistas_ucatolica/index.php/RevArq/article/view/449/1381\#r27

Torres, E. (2009). Apropiación versus usos del espacio público. La ciudad viva. Recuperado de http://www.laciudadviva.org/blogs/?p=3465

Urban 95. (2018). Urban 95-Lima Norte. Banco de desarrollo de América Latina. Recuperado de https://www.caf.com/media/9287200/5-concurso-desarro llo-urbano-primer-premio-urban-95.pdf

Ujang, N. y Zakariya, K. (2015). The notion of place, place meaning and identity in urban regeneration. Procedia-Social and behavioral sciences. Recuperado de https://doi.org/10.1016/j.sbspro.2015.01.073

Uría, J. (2001). Lugares para el ocio. Espacio público y espacios recreativos en la Restauración española. Historia Social. Recuperado de http://www.jstor. org/stable/40340787 